\title{
Perfil epidemiológico e alterações anatomopatológicas de biópsias de rins esquerdos de sete cães acometidos por Dioctophyme renale em rim direito
}

\author{
Epidemiological profile and anatomopathological changes of biopsies of left kidneys from seven \\ dogs affected by Dioctophyme renale in right kidney \\ Perfil epidemiológico y cambios anatomopatológicos de biopsias de riñón izquierdo de siete perros \\ afectados por Dioctophyme renale en riñón derecho
}

\author{
Aline Xavier Fialho Galiza \\ ORCID: https://orcid.org/0000-0002-1139-7117 \\ Universidade Federal de Pelotas, Brasil \\ E-mail: aline.xavfialho@hotmail.com \\ Luísa Mariano Cerqueira da Silva \\ ORCID: https://orcid.org/0000-0001-6158-4204 \\ Universidade Federal de Pelotas, Brasil \\ E-mail: luisamarianovet@yahoo.com.br \\ Luísa Grecco Correa \\ ORCID: https://orcid.org/0000-0003-1355-2907 \\ Universidade Federal de Pelotas, Brasil \\ E-mail: luisagcorrea@gmail.com \\ Eduardo Gonçalves \\ ORCID: https://orcid.org/0000-0001-8951-9316 \\ Universidade Federal de Pelotas, Brasil \\ E-mail: goncalves-eduardo@outlook.com \\ Aline do Amaral \\ ORCID: https://orcid.org/0000-0003-3039-3194 \\ Universidade Federal de Pelotas, Brasil \\ E-mail: amaralaaline@gmail.com \\ Pâmela Caye \\ ORCID: https://orcid.org/0000-0002-6456-8100 \\ Universidade Federal de Santa Maria, Brasil \\ E-mail: pamiscaye@gmail.com \\ Júlia Vargas Miranda \\ ORCID: https://orcid.org/0000-0001-6576-3496 \\ Universidade Federal de Pelotas, Brasil \\ E-mail: juvm@live.com \\ Clarissa Caetano de Castro \\ ORCID: https://orcid.org/0000-0002-4822-034X \\ Universidade Federal de Pelotas, Brasil \\ E-mail: clarissac.decastro@gmail.com \\ Josaine Cristina da Silva Rappeti \\ ORCID: https://orcid.org/0000-0002-8822-0387 \\ Universidade Federal de Pelotas, Brasil \\ E-mail: josainerappeti@yahoo.com.br \\ Thomas Normanton Guim \\ ORCID: https://orcid.org/0000-0001-8278-1397 \\ Universidade Federal de Pelotas, Brasil \\ E-mail: thomasguim@ @otmail.com \\ Cristina Gevehr Fernandes \\ ORCID: https://orcid.org/0000-0002-0729-2099 \\ Universidade Federal de Pelotas, Brasil \\ E-mail: crisgevf@yahoo.com.br \\ Fabiane Borelli Grecco \\ ORCID: https://orcid.org/0000-0002-3996-315X \\ Universidade Federal de Pelotas, Brasil \\ E-mail: fabianegrecco18@gmail.com
}

\section{Resumo}

A dioctofimatose é uma doença causada pelo nematódeo Dioctophyme renale, que acomete principalmente rins direitos de cães e ao penetrar na cápsula renal causa destruição e atrofia do parênquima, podendo restar apenas uma 
cápsula fibrosa do rim afetado. Dessa forma, buscando estabelecer as condições histológicas do rim contralateral (RCL) dos animais acometidos pela parasitose, o objetivo desse estudo foi descrever o perfil epidemiológico e alterações anatomopatológicas encontradas em biópsias de rins esquerdos de sete cães submetidos a nefrectomia em decorrência de parasitismo por Dioctophyme renale em rim direito. Informações referentes à raça, sexo, faixa etária, histórico clínico, e alterações histológicas, foram tabulados e avaliados. Para as biópsias foi utilizado o método incisional. Microscopicamente em três amostras (3/7) havia glomerulonefrite membranosa, em quatro (4/7) observouse infiltrado inflamatório de células mononucleadas e fibrose focal em duas amostras analisadas (2/7). Em uma amostra havia glomeruloesclerose segmentar focal e em outra não havia alteração histológica. Foram contabilizadas mais de uma alteração em uma única amostra. As alterações encontradas nas biópsias indicam algum comprometimento do rim remanescente e sugerindo que possa haver ação sistêmica, ou pelo menos inter-renais das enzimas esofágicas produzidas pelo parasito Este estudo espera contribuir para o estadiamento das funções renais do rim remanescente e auxiliar no estabelecimento do tratamento em cada caso.

Palavras-chave: IRA; Afecção renal; Glomerulonefrite.

\begin{abstract}
Dioctofimatosis is a disease caused by the nematode Dioctophyme renale, which occurs mostly in dogs and has predilection mostly for the right kidney and when penetrating the renal capsule, it causes destruction and atrophy of the renal parenchyma, and only one fibrous capsule of the affected kidney may remain. Thus, seeking to establish the histological conditions of the contralateral kidney (RCL) of the affected animals, the objective of this study was to describe the epidemiological profile and anatomopathological changes found in left kidney biopsies of seven dogs undergoing nephrectomy due to Dioctophyme renale parasitism in right kidney. Information relative to race, sex, age range, medical history, histological changes, was tabbed and evaluated. For the biopsies were used incisional method. Of the 10 samples, 7 biopsy samples, collected by the incisional technique, were satisfactory for the study. Microscopically there were three cases (3/7) with membranous glomerulonephritis, four (4/7) had inflammatory infiltrate of mononucleated cells and focal fibrosis in two analyzed samples (2/7). In one case (1/7) focal segmental glomerulosclerosis was observed and one sample didn't show any histological changes. It may have more than one change in one sample. The changes found in the biopsies indicate some impairment of the remaining kidney and suggesting that there maybe systemic, or at least inter-renal, action of the esophageal enzymes produced by the parasite. This study hopes to contribute to the staging of the renal functions of the remaining kidney and assist in the establishment of treatment in each case.
\end{abstract}

Keywords: AKI; Renal disease; Glomerulonehritis.

\begin{abstract}
Resumen
La dioctofimatosis es una enfermedad causada por el nematodo Dioctophyme renale, que afecta principalmente al riñón derecho de los perros y cuando penetra en la cápsula renal provoca la destrucción y atrofia del parénquima renal, dejando sólo una cápsula fibrosa del riñón afectado. Así, buscando establecer las condiciones histológicas del riñón contralateral (LCR) de los animales afectados, el objetivo de este estudio fue describir el perfil epidemiológico y los cambios anatomopatológicos encontrados en biopsias de riñón izquierdo de siete perros sometidos a nefrectomía por parasitismo por Dioctophyme renale en riñón derecho. Se tabuló y evaluó la información relativa a la raza, el sexo, el rango de edad, la historia clínica y los cambios histológicos. Para las biopsias se utilizó el método incisional. Microscópicamente tres casos (3/7) presentaban glomerulonefritis membranosa, cuatro (4/7) presentaban infiltrado inflamatorio de células mononucleadas y fibrosis focal en dos muestras analizadas (2/7). Em uma observó glomeruloesclerosis segmentaria focal en un caso (1/7) y una muestra no presentaba ninguna alteración histológica. Puede haber más de una alteración y una muestra. Los cambios encontrados en las biopsias indican alguna alteración del riñón remanente y sugieren que puede haber una acción sistémica, o al menos interrrenal, de las enzimas esofágicas producidas por el parásito. Este estudio espera contribuir a la estadificación de las funciones renales del riñón restante y ayudar a establecer el tratamiento en cada caso.
\end{abstract}

Palabras clave: IRA; Enfermidad renal; Glomerulonefritis.

\title{
1. Introdução
}

A dioctofimatose é causada pelo nematódeo Dioctophyme renale, que parasita principalmente os animais domésticos e silvestres (Souza et al., 2019). A doença é uma zoonose, que pode parasitar tecido subcutâneo e rins de humanos (Souza et al., 2019). Geralmente os parasitos são encontrados em rins direitos, porém há descrição de parasitismo em próstata, cavidade abdominal e até mesmo bilateral (Sapin et al., 2017b; Caye et al., 2020, Butti et al., 2020). Dentre os hospedeiros definitivos o cão é o animal doméstico mais acometido (Leite et al., 2005). O ciclo biológico do parasita tem como hospedeiros intermediários peixes de água doce, anelídeos aquáticos e rãs, que são ingeridos pelos hospedeiros definitivos, geralmente carnívoros domésticos e selvagens (Mayrink et al. 2000). Os parasitos ingeridos migram da parede intestinal para o rim, com 
predileção pelo rim direito, causando uma destruição progressiva das camadas cortical e medular renal, restando somente uma cápsula fibrosa (Zabott et al., 2012).

O diagnóstico de dioctofimatose ocorre por meio de achados incidentais dos helmintos em necrópsias, procedimentos cirúrgicos, exames de ultrassonografia abdominal e a presença de ovos ou nematódeos no exame de urina (Perera et al., 2017). Os tratamentos de rotina são a nefrotomia e a nefrectomia do órgão acometido, juntamente com a remoção cirúrgica do parasito (Souza et al., 2019).

O uso da biópsia renal em cães e gatos vem crescendo na medicina veterinária (Tabet et al., 2005). A biópsia renal pode determinar diagnósticos definitivos, estadiamento de injúrias renais, escolha de tratamentos (Hernandéz, 2009), e se tornam valiosas ferramentas de diagnóstico e prognóstico (Rezaie, 2008). Dessa forma, a biópsia renal, quando assim indicada, permite que o patologista estabeleça o diagnóstico histológico a fim de realizar o estadiamento das funções renais do paciente (Lane, 1994). Portanto, a realização de biópsia do rim contralateral (RCL) ao acometido por Dioctophyme renale, é um procedimento que poderá fornecer subsídios e informações inerentes a conduta terapêutica do paciente.

Nesse contexto, este estudo teve como objetivo descrever o perfil epidemiológico e avaliar as condições histológicas do rim contralateral (RCL) de sete animais acometidos por Dioctophyme renale em rim direito, atendidos pelo Serviço de Oncologia Veterinária da Universidade Federal de Pelotas (SOVET-UFPel), no período de abril de 2019 a agosto de 2020.

\section{Metodologia}

Durante o período de abril de 2019 a agosto de 2020 foram avaliadas biópsias renais provenientes de pacientes que viviam sob cuidados de uma ONG (organização não governamental) localizado na região metropolitana de Pelotas e que recebem cuidados veterinários no Hospital de Clínica Veterinária da FV/UFPel. Foram avaliadas sete biópsias de rins contralaterais aos acometidos por dioctofimatose, as quais foram obtidas durante o tratamento cirúrgico por nefrectomia do rim afetado. As biópsias dos rins contralaterais. foram enviadas para análise anatomopatológica no SOVET-UFPel. Também, foram coletadas informações dos protocolos de encaminhamento, referentes a dados demográficos e epidemiológicos, como raça, sexo, faixa etária e histórico clínico (Tabela 1). Quanto à raça, os animais foram classificados como "com raça definida" (CRD) e "sem raça definida" (SRD). Quanto a faixa etária, o método utilizado foi o determinado por Fighera, et al. (2008) que classifica como filhotes (menores de um ano), adultos (de um a nove anos) e idosos (acima de 9 anos). 
Tabela 1. Dados epidemiológicos de 7 cães parasitados por Dioctophyme renale e alterações histopatológicas encontradas em biópsias realizadas no período de abril de 2019 a agosto de 2020.

\begin{tabular}{l|l|l|l|l|l}
\hline Paciente & Raça & Sexo & $\begin{array}{l}\text { Faixa } \\
\text { etária }\end{array}$ & Histórico clínico & Achados microscópicos \\
\hline $\mathbf{1}$ & CRD & Macho & Adulto & $\begin{array}{l}\text { Nódulo no } \\
\text { subcutâneo no } \\
\text { flanco direito }\end{array}$ & Tecido renal esquerdo, não apresenta alteração. \\
\hline $\mathbf{2}$ & SRD & Fêmea & Idosa & $\begin{array}{l}\text { Claudicação de } \\
\text { membros pélvicos }\end{array}$ & $\begin{array}{l}\text { Tecido renal esquerdo, amostra eosinofílica, } \\
\text { apresentando infiltrado inflamatório intersticial } \\
\text { de células mononucleadas, focos hemorrágicos( } \\
\text { da biópsia) Apresenta um pouco de fibrose } \\
\text { intersticial e espessamento da cápsula } \\
\text { glomerular. }\end{array}$ \\
\hline $\mathbf{3}$ & SRD & Fêmea & Adulta & Sem histórico & $\begin{array}{l}\text { Tecido de rim esquerdo, apresentando focos } \\
\text { hemorrágicos, infiltrado inflamatório } \\
\text { mononuclear e regiões de fibrose. }\end{array}$ \\
\hline $\mathbf{4}$ & SRD & Fêmea & Adulta & Hematúria & $\begin{array}{l}\text { Tecido renal esquerdo, amostra bem } \\
\text { eosinofílica, acidofílica e com muito artefato. } \\
\text { Tecido com infiltrado inflamatório difuso. }\end{array}$ \\
\hline $\mathbf{5}$ & SRD & Fêmea & Idoso & Atropelamento & $\begin{array}{l}\text { Tecido renal esquerdo, amostra eosinofílica, } \\
\text { apresenta foco hemorrágico, infiltrado } \\
\text { inflamatório de células mononucleadas focal, } \\
\text { glomeruloesclerose. }\end{array}$ \\
\hline $\mathbf{7}$ & SRD & Fêmea & Adulta & Fem histórico & $\begin{array}{l}\text { Tecido renal esquerdo, apresentando } \\
\text { espessamento da cápsula glomerular. }\end{array}$ \\
\hline
\end{tabular}

Fonte: Autores.

Os animais foram submetidos a biópsia simultaneamente a retirada do rim afetado. A técnica incisional em cunha, foi realizada sendo seccionado inicialmente apenas a cápsula e depois a incisão no parênquima renal. Imediatamente após, foi realizada a síntese do tecido com o ponto, em Wolf com fio nylon 2-0, já previamente montado, evitando assim hemorragias. Os fragmentos de órgãos foram fixados em formalina tamponada 10\%, processadas rotineiramente e coradas com Hematoxilina e Eosina, para a realização do estudo histopatológico.

\section{Resultados}

Dos cães com Dioctofimatose desse estudo, a maioria tratava-se de cães errantes que foram resgatados pela prefeitura de Pelotas e, devido a isso, não se obteve o histórico clínico de três animais. Quanto ao gênero sexual, obteve-se seis cães fêmeas e um macho. A doença predominou em animais adultos 4/7 (57,1\%), seguido de cães idosos 2/7 (28,5\%) e em $1 / 7$ $(14,2 \%)$ não havia informação da idade. Em relação às raças, a parasitose afetou um animal da raça labrador e seis animais sem raça definida $(\mathrm{SRD})$.

A técnica de biopsia incisional foi a escolhida levando-se em consideração a maior praticidade para execução e a coleta de um fragmento adequado para avaliação. A avaliação histológica (Figura 1) demonstrou glomerulonefrite membranosa em três casos (3/7-43\%). Em quatro casos (4/7-57\%), observou-se infiltrado inflamatório de células mononucleadas e fibrose focal foi observada em duas amostras analisadas (2/7 28,5\%). Em um caso, ainda, foi observado glomeruloesclerose segmentar focal (1/7-1\%). Notadamente, uma mesma amostra podia exibir mais de uma alteração. Em nenhuma amostra foram observadas alterações indicativas de hipertrofia compensatória renal. 
Figura 1. Biópsias renais contralaterais de cães parasitados por D. renale. a) e b) Infiltrado inflamatório mononuclear em aumento de 10x e 40 x respectivamente. c) Glomerulonefrite membranosa em aumento de 40x. Coloração de H\&E.

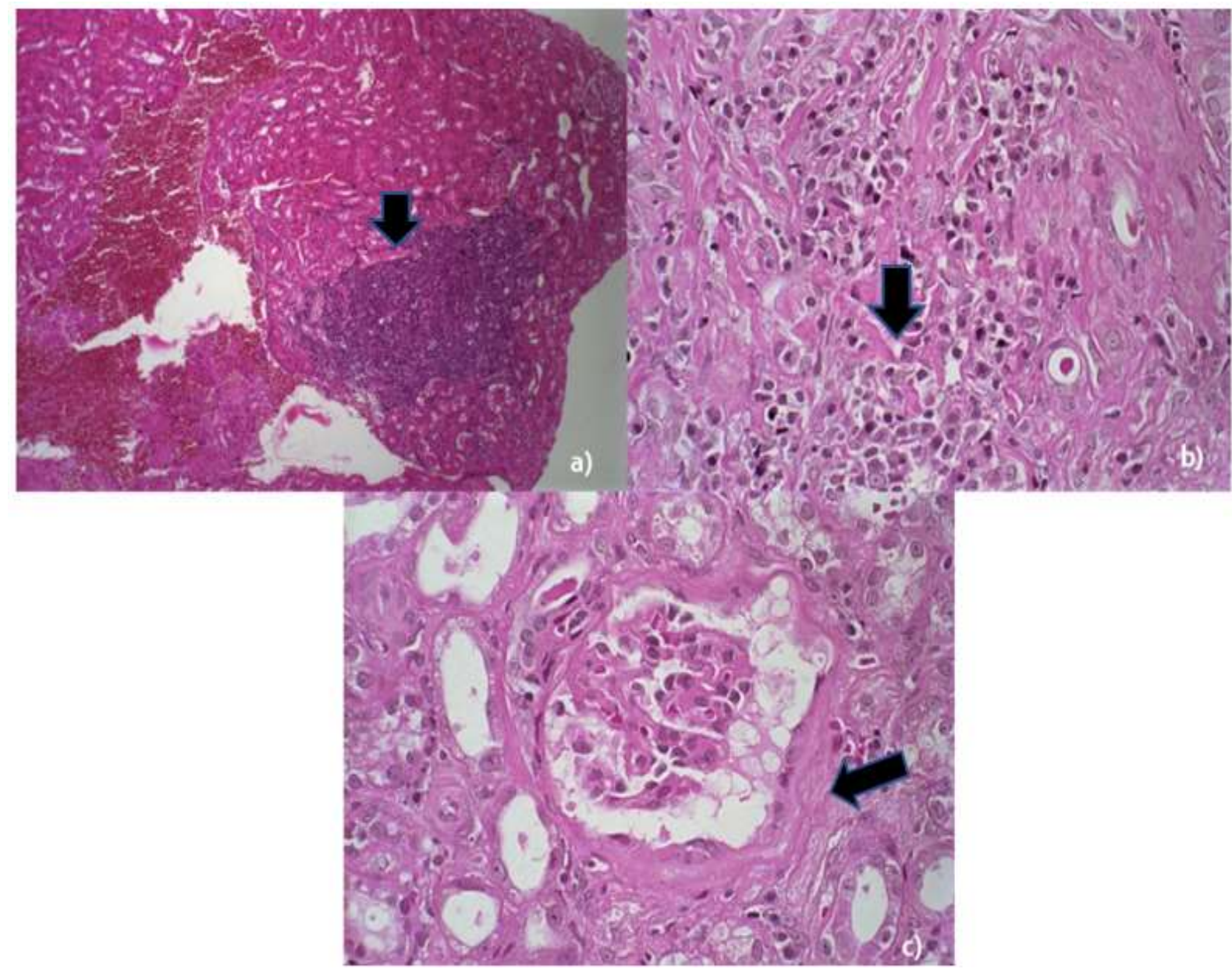

Fonte: Arquivo pessoal (2020).

\section{Discussão}

As amostras analisadas pertenciam em sua maioria à cães errantes ou com livre acesso as ruas, que são citados na literatura como principais acometidos pelo D.renale (Leite et al., 2005). O diagnóstico presuntivo de dioctofimatose e a indicação de nefrectomia/nefrotomia em geral são estabelecidos através da ultrassonografia, o que demonstra que a doença possa ser subclínica até que os danos do rim afetado não possam mais ser reparados e/ou quando o rim contralateral não consiga mais suprir as funções do sistema urinário (Sapin et al., 2017). Alguns autores citam que imagens ultrassonográficas de rins contralaterais podem exibir um aumento de volume em alguns casos relatados (Costa et al., 2004; Oliveira et al., 2005). No presente trabalho todos os animais tiveram o diagnóstico confirmado pelo exame ultrassonográfico.

Todas as biopsias foram realizadas em RCL esquerdos, e os sete pacientes tinham o rim direito acometido pela parasitose em concordância com Zabott e colaboradores, (2012). Nos casos avaliados, o Dioctophyme renale parasitou principalmente fêmeas adultas sem raça definida, embora o parasitismo não tenha predileção por sexo ou raça (Pereira et al., 2006).

A severidade da lesão renal causada pelo D. renale, depende do número de parasitas que afetam o rim, da duração da infecção, do número de rins envolvidos e da presença ou ausência de doença renal concomitante (Freire et al., 2002). Em nosso estudo os animais tiveram indicação para nefrectomia devido ao grande comprometimento do rim afetado, por esse motivo também foram escolhidos para a realização da biópsia contralateral, com objetivo de estadiar a função renal do rim 
remanescente. As lesões microscópicas mais comumente descritas na dioctofimatose são proliferação de tecido conjuntivo fibroso associado à nefrite intersticial mononuclear, com atrofia do parênquima renal, degeneração tubular e glomeruloesclerose (Milanelo et al. 2009, Sapin et al. 2017).

A glomerulopatia é considerada uma causa comum e importante de doença renal crônica (DRC) (Grauer, 2005; Vaden, 2011), que pode causar alterações morfológicas no glomérulo como proliferação de células e matriz mesangial e espessamento da membrana basal glomerular, podendo também progredir para glomeruloesclerose (Grant, 2001; Grauer, 2005). A presença de infiltrado inflamatório de células mononucleadas, glomerulonefrite membranosa, glomeruloesclerose segmentar focal e fibrose focal, indicam que o animal já possuía alterações que podiam evoluir e comprometer a função desse rim remanescente, associado ao fato de que todas essas alterações são encontradas comumente em rins parasitados, sugerindo que possa haver ação sistêmica, ou pelo menos inter-renais das enzimas esofágicas produzidas pelo parasito que são a principal forma de agressão ao parênquima renal.

\section{Considerações Finais}

Podemos concluir que nas biópsias analisadas foram encontradas alterações histopatológicas que evidenciavam algum grau de comprometimento do rim remanescente, reforçando a importância de conhecer as condições histológicas do rim contralateral (RCL) dos animais acometidos por D. renale para estabelecer o estadiamento das funções renais do paciente e para a determinação do prognóstico contribuindo para um tratamento adequado em cada caso.

\section{Referências}

Butti, M. J., Gamboa, M., Terminiello, J., Urbiztondo, M., Polizzi, C., Carina, F., \& Radman, N. (2020) Dioctofimatosis renal, abdominal e intraprostática en um canino. Revista Argentina de Parasitologia. Vol. 9 № 1.

Caye, P., Schmitt, T., Cavalcanti, G., \& Rappeti, J. C. (2020) Prevalência de Dioctophyme renale (Goeze, 1782) em cães de uma organização não governamental do sul do Rio Grande do sul - Brasil. Archives of Veterinary Science .25, 2,46-55.

Costa, P. R. S., Argolo, Neto N. M., Oliveira, D. M. C., Vasconcellos, R. S., \& Menezes, F. M. (2004). Dioctofimose e leptospirose em um cão - relato de caso. Revista Clínica Veterinária. São Paulo, 51, 48-50.

Fighera, R. A., Souza, T. M., Silva, M. C., Brum, J. S., Graça, D. L., Kommers, G. D., Irigoyen, L. F., \& Barros, C. S. L. (2008). Causas de morte e razões para eutanásia de cães da Mesorregião do Centro Ocidental Rio-Grandense (1965-2004). Pesquisa Veterinária Brasileira. 28(4):223-230, abril de 2008.

Freire, S. E., Fedozzi, F., Freire, A. F., Monteiro Júnior, L. A., \& Navarro, S. (2002). Dioctophyme renale em cão: relato de caso. In: III Encontro de produção acadêmica medicina veterinária FEOB. São João da Boa Vista, Anais. São João da Boa Vista: FEOB, 174 - 177.

Grant, D. C., \& Forrester, S. (2001). Glomerulonephritis in dogs and cats: Glomerular function, pathophysiology, and clinical signs. Compendium on Continuing Education for the Practicing Veterinarian, 23(8), 739- 747.

Grauer, G. F. (2005). Canine glomerulonephritis: New thoughts on proteinuria and treatment. Journal of Small Animal Practice, 46(10), 469-478.

Hernandéz, F. R. (2009). Biopsia renal. Acta Pediátrica de México, [s. 1.], 30(1), 36-53.

Lane, I. F., Grauer, G. F., \& Fettman, M. J. (1994). Acute renal failure. Part II. Diagnosis, management, and prognosis. Compend Contin Educ Vet.; 16:625-45.

Leite, L. C., Círio, S. M., Diniz, J. M. F., Luz, E., Navarro-Silva, M. A., Silva, A. W. C. Leite, S. C., Zadorosnei, A. C., Musiat, K. C., Veronesi, E. M., \& Pereira, C. C. (2005). Lesões anatomopatológicas presentes na infecção por Dioctophyma renale (Goeze, 1782) em cães domésticos (canis familiaris,linnaeus, 1758). Archives of Veterinary Science, Paraná, 10(1), 95-101.

Mayrink, K. C., Paes-de-Almeida, E. C., \& Thomé, S. M. G. (2000). Dioctophyma renale (GOEZE, 1782) em cães. Caderno Técnico Científico da Escola de Medicina Veterinária da Universidade do Grande Rio, Rio de Janeiro, (2), 20-40.

Milanelo, L., Moreira, M. B., Fitorra, L. S., Petri, B. S. S., Alves, M., \& Santos, A. C. (2009). Occurrence of parasitism by Dioctophyma renale in ring-tailed coatis (Nasua nasua) of the Tiete Ecological Park, São Paulo, Brazil. Pesq. Vet. Bras. 29(12):959-962.

Oliveira, L. L., Attallah, F. A., Santos, C. L., Wakofs, T. N., Rodrigues, M. C. D., \& Santos, A. E. (2005). O uso da ultrassonografia para o diagnóstico de Dioctophyma renale em cão - relato de caso. Revista Universidade Rural, Seropédica, 25, suplemento, 323-324.

Pereira, B. J., Girardelli, G. L., Trivilin, L. O., Lima, V. R., Nunes, L. C., \& Martins, I. V. F. (2006). Ocorrência de Dioctofimose em cães do município de Cachoeiro do Itapemirim, Espírito Santo, Brasil, no período de maio a dezembro de 2004. Revista Brasileira de Parasitologia Veterinária, Rio de Janeiro, 15(3), 123-125. 
Research, Society and Development, v. 10, n.6, e50310615703, 2021

(CC BY 4.0) | ISSN 2525-3409 | DOI: http://dx.doi.org/10.33448/rsd-v10i6.15703

Perera, S. C., Rappeti, J. C. S., Milech, V., Braga, F. A., Cavalcanti, G. A. O., Nakasu, C. C., Durante, L., Vives, P., \& Cleff, M. B. (2017). Eliminação de Dioctophyme renale pela urina em canino com dioctofimatose em rim esquerdo e cavidade abdominal - primeiro relato de caso no Rio Grande do Sul. Arquivo Brasileiro de Veterinária e Zootecnia, [s. 1.], 69(3), 618-622.

Rezaie, A., Mousavi, G., Mohajeri, D., \& Asadnasab, G. (2008). Complicatios of the ultrasound-guided needle biopsy of the kidney in dogs. J Anim Vet Adv. 7(10):1207-13.

Sapin, C. F., Silva-Mariano, L. C., Piovesan, A. D., Fernandes, C. G., Rappeti, J. C., Braga, F. A. V., Cavalcante, G. A., Rosenthal, B. M., \& Grecco, F. B. (2017). Estudo anatomopatológico de rins parasitados por Dioctophyme renale em cães. Acta Scientiae Veterinariae, 45, 1-7.

Sapin, C. F., Silva-Mariano, L., Grecco-Corrêa, L., Rappeti, J. C. S., Durante, L., Perera, S. C., Cleff, M. B., \& Grecco, F. B. (2017b). Dioctofimatose renal bilateral e disseminada em cão. Pesquisa Veterinária Brasileira, 37(12), 1499-1504.

Souza, M. S. D., Duarte, G. D., Brito, S. A. P. D., \& Farias, L. A. D. (2019). Dioctophyma renale: Revisão. Pubvet, [S. l.], 13(6), a.346, 1-6, 25 jun.

Tabet, A. F. (2005) Comparação entre duas técnicas de biópsia renal guiadas por laparoscopia em eqüinos. Brazilian Journal of Veterinary Research and Animal Science, [s. 1.], 42(2), 150.

Vaden, S. L. (2011) Glomerular Disease. Topics in Companion Animal Medicine, 26(3), 128-134.

Zabott, M. V., Pinto, S. B., Viott, A. M., Tostes, R. A., Bittencourt, L. H. F. B., Konell, A. L., \& Gruchouskei, L. (2012). Ocorrência de Dioctophyma renale em Galictis cuja. Pesquisa Veterinária Brasileira, [s. l.], p. 786-788. 